\title{
Single-Nucleotide Polymorphisms Related to Leprosy Risk and Clinical Phenotypes Among Chinese Population
}

\author{
Si-Yu Long' \\ Le Wang ${ }^{1,2}$ \\ Hai-Qin Jiang' \\ Ying Shi (D) \\ Wen-Yue Zhang' \\ Jing-Shu Xiong' \\ Pei-Wen Sun ${ }^{1,2}$ \\ Yan-Qing Chen' \\ You-Ming Mei iD \\ Chun Pan' \\ Gai Ge' \\ Zhen-Zhen Wang' \\ Zi-Wei Wu' \\ Mei-Wen Yu ${ }^{1,2}$ \\ Hong-Sheng Wang ${ }^{1-3}$ \\ 'Jiangsu Key Laboratory of Molecular \\ Biology for Skin Diseases and STIs, \\ Institute of Dermatology, Chinese \\ Academy of Medical Sciences and Peking \\ Union Medical College, Nanjing, 210042, \\ People's Republic of China; ${ }^{2}$ National \\ Centre for Leprosy Control, China CDC, \\ Nanjing, People's Republic of China; \\ ${ }^{3}$ Centre for Global Health, School of \\ Public Health, Nanjing Medical University, \\ Nanjing, People's Republic of China
}

Correspondence: Hong-Sheng Wang; Mei-Wen Yu

Jiangsu Key Laboratory of Molecular Biology for Skin Diseases and STIs, Institute of Dermatology, Chinese Academy of Medical Sciences and Peking Union Medical College, St 12

Jiangwangmiao, Nanjing, Jiangsu, 210042,

People's Republic of China

Email whs33@vip.sina.com;

yumeiwen@163.com
Background: Genome-wide association studies (GWASs) have identified some immunerelated single-nucleotide polymorphisms (SNPs) to be associated with leprosy.

Methods: This study investigated the association of 17 SNPs based on previously published GWAS studies with susceptibility to leprosy, different polar forms and immune states of leprosy in a case-control study from southwestern China, including 1344 leprosy patients and 2732 household contacts (HHCs) (1908 relatives and 824 genetically unrelated contact individuals). The differences of allele distributions were analyzed using chi-squared analysis and logistic regression.

Results: After adjusting covariate factors, rs780668 and rs3764147 polymorphisms influenced susceptibilities to genetically related or unrelated leprosy contact individuals. rs142179458 was associated with onset early cases, rs73058713 A allele and rs3764147 A allele increased the risk of reversal reaction, while rs3764147 G allele had higher risk to present lepromatous leprosy and erythema nodosum leprosum.

Conclusion: Our results demonstrated that genetic variants in the LACC1, HIF1A, SLC29A3 and $C D H 18$ genes were positively correlated with the occurrence of leprosy and leprosy clinical phenotypes, providing new insights into the immunogenetics of the disease.

Keywords: leprosy, association study, susceptibility gene, polymorphisms

\section{Introduction}

Leprosy, which is a chronic infectious disease caused by intracellular pathogen Mycobacterium leprae (M. leprae), remains endemic in many areas in Asia and south America. In China, the overall leprosy incidence is still at a relatively low endemic level, mainly in southwestern provinces, including Sichuan, Hunan, Yunnan and Guizhou. ${ }^{1}$

It is estimated that only a small fraction of individuals exposed to M. leprae are successfully infected. ${ }^{2}$ The complex interplay between host and pathogens may be one of the explanations for such variability, which is largely influenced by the genetic make-up of the host. ${ }^{3}$ A prospective cohort study demonstrated that genetic relationship was a relevant and independent risk factor for leprosy. ${ }^{4}$ The genetic factors may influence host's immunologic status and determine the infection outcomes. Leprosy ranges from a form with a robust immune response and very few organisms (tuberculoid or paucibacillary) to a form with a weaker immune response and a higher burden of organisms (lepromatous or multibacillary), which is a good model for studying the genetic basis and immune response of chronic infection. ${ }^{5}$ 
The tuberculoid leprosy patients present strong cellular (Th1) immune response with increased production of proinflammatory cytokines, on the other hand, lepromatous leprosy is characterized by a predominantly antibody based (Th2) immune response. Leprosy patients may develop leprosy reactions during the medical course, which is the major cause of permanent neural damage with consequent disabilities, including Type 1 or Reversal Reaction (RR) and Type 2 or Erythema nodosum leprosum (ENL). ${ }^{6}$ It is widely regarded as an immune complex disorder, and some proinflammatory cytokines have been observed; however, their roles in pathogenesis remain unclear. $^{7}$

Evidence from GWASs ${ }^{8-14}$ suggests that host genetic factors might contribute to the leprosy per se, disease clinical forms and the occurrence of leprosy reactions. Among the studies, several genes are immune-related. SNPs located in RAB32, HIF1A, BATF3, LACC1, CTSB, TNFSF15, CDH18, SLC29A3, DEC1, FLG, NOD2, IL18RAP/IL18R1, NCKIPSD and CARD9 were significantly associated with leprosy. Other association studies have replicated these results in different independent populations. ${ }^{15-19}$

As during infection and inflammation, genetic factors were linked with important immunological consequences and leprosy clinical manifestations vary depending on the host's ability to mount an acquired immune response to infection. In this study, we utilized published genetic risk variants in GWAS with case-control study and tried to evaluate the association of genetic polymorphisms with leprosy development genetic predisposition in Chinese population, which is helpful for medical workers to construct genetic risk models for tracing leprosy higher-risk HHCs and identify the predictive markers of leprosy reactions.

\section{Patients and Methods Study Participants}

We performed a case-control study involving individuals from January 2010 to June 2014 in four provinces (Yunnan, Guizhou, Sichuan and Hunan). A total of 1466 leprosy patients and 2904 healthy leprosy HHCs were enrolled. Patients with leprosy registered in 2010-2014 in the study area were selected as the case group and classified according to initial clinical evaluation based on clinical manifestations, slit skin smears and histopathological examinations. The control group was composed of healthy leprosy HHCs from the same geographic region including 2029 relatives (1663 first-, 335 second-, and 31 third-degree family members) and 875 genetically unrelated contact individuals (spouses). HHCs were defined as people living under the same roof and sharing food with the patient for at least six months among the past six years. The control group excluded those who refused to provide informed consent, any person that received treatment for tuberculosis or leprosy within one year.

Blood samples were collected from all participating subjects after obtaining an informed written consent. All participating individuals provided personal data including sex, age and ethnicity. Data such as age at diagnosis, leprosy classifications and reactional states were collected from medical record.

\section{SNP Selection and Genotyping}

Based on the previously published GWAS studies and one study combined whole-exome sequencing and targeted next-generation sequencing within the GWAS loci, ${ }^{8-14}$ we selected 17 SNPs wherein a genome-wide significant association $\left(P<5 \times 10^{-8}\right)$ between the SNPs in the genes RAB32, HIF1A, BATF3, LACC1, CTSB, TNFSF15, CDH18, SLC29A3, DEC1, FLG, NOD2, IL18RAP/ IL18R1, NCKIPSD, CARD9 and leprosy (S1 Table). Based on multiplex polymerase chain reaction (PCR) to precisely genotype SNPs with next-generation sequencing. After the PCR amplification, the products genotyped according to the manufacturer's protocol using the Illumina HiSeq X-10 platform. The adapter sequences were trimmed out, and we removed the reads which $\mathrm{N}$ content in single-ended sequencing read was more than $10 \%$ of the read length ratio, and the low quality (Qphred $\leq 20)$ base number in single-ended sequencing read was more than $50 \%$ of the read length ratio. Clean sequence reads were aligned to the reference GRCH38 by Burrows-Wheeler Aligner (http://bio-bwa.sourceforge. net/). ${ }^{20}$ SNP calling was performed by GATK (Genome Analysis Toolkit, https://www.broadinstitute.org/gatk/). ${ }^{21}$ We excluded SNPs and individuals with genotype call rates $<95 \%$, SNPs with minor allele frequency (MAF) $<1 \%$, and Hardy-Weinberg Equilibrium $\mathrm{P}<1.0 \times 10^{-3}$ in controls. Ultimately, two variants with MAF $<1 \%$ (rs149308743 and rs145562243) and one variant HardyWeinberg Equilibrium $\mathrm{P}<1.0 \times 10^{-3} \quad(\mathrm{rs} 2221593)$ were eliminated.

\section{Statistical Analysis}

Cases and controls (all HHCs, heredity-related contacts, first-degree family members, non-Heredity-related 
contacts of leprosy cases) were compared according to allele with the Pearson $\chi^{2}$ test or Fisher test using PLINK v 1.07. P value was considered to be statistically significant at the 0.05 level. We also analyze the differences in distributions of alleles between different leprosy clinical phenotypes (patients diagnosed at the age $\leq 14$ year vs >14-year; paucibacillary leprosy cases vs multibacillary leprosy cases; tuberculoid cases vs lepromatous cases; RR in BT, BB and BL leprosy cases vs BT, BB and $\mathrm{BL}$ leprosy cases free of RR; ENL in BL and LL leprosy cases vs BT, BB and BL leprosy cases free of ENL; ENL vs RR). Meanwhile, we investigated the associations between leprosy and clinical phenotypes with SNPs based on a logistic regression model adjusted for age, ethnicity and gender.

\section{Results}

\section{Baseline Characteristics of Participations}

A total of 294 individuals with genotype call rates $<95 \%$ were eliminated. Ultimately, a total of 1344 leprosy patients (711 Han Chinese, 936 males, 408 females, with a mean age of $44.05 \pm 15.80$ years), 1908 relatives (1560 first-, 318 second-, and 30 third-degree family members, 1106 Han Chinese, 1123 males, 785 females, with a mean age of $34.17 \pm 15.54$ years) and 824 genetically unrelated contact individuals (453 Han Chinese, 248 males, 576 females, with a mean age of $46.57 \pm 12.81$ years) were recruited. S2 Table shows the distribution of ethnicity. The baseline characteristics of participants are listed in Table 1. Significant difference was found in mean age, ethnical and sex ratio between leprosy patients and controls.

\section{Association Between Leprosy and SNPs}

Allele distributions between leprosy cases and HHCs were analyzed using $\chi 2$ test or Fisher test, $\underline{\mathrm{S} 3}$ and $\underline{\mathrm{S} 4 \text { Tables }}$ showed the distributions of alleles and genotypes for the study subjects. The allele distributions of rs6478108 at TNFSF 15 locus, rs663743 at CCDC88B locus, rs780668 at the SLC29A3 locus, rs9302752 at the NOD2 locus and rs3764147 at the $L A C C 1$ locus were significantly different between patients and HHCs. Four variants showed an association at $P<0.05$ after adjusting for sex, ethnicity and age in the logistic regression, including rs780668 $(\mathrm{P}<0.001), \operatorname{rs} 6478108(\mathrm{P}=0.02)$, rs9302752 $(\mathrm{P}=0.003)$ and rs3764147 $(\mathrm{P}<0.001)$. The characteristics and association results of the 14 variants are displayed in Table 2.
The allele distributions of rs6478108, rs780668 and rs3764147 were significantly different between patients and healthy relatives control group. Two variants showed an association at $\mathrm{P}<0.05$ after adjusting for confounding factors, including rs780668 $(\mathrm{P}<0.001)$ and $\mathrm{rs3764147}$ $(\mathrm{P}<0.001)$. Comparing with the first-degree family members, the allele distributions of rs780668 $(\mathrm{P}<0.001)$ and rs3764147 $(\mathrm{P}<0.001)$ also showed significantly different.

Comparing with the genetically unrelated contact individuals, the allele distributions of rs9302752, rs6478108, rs780668 and rs3764147 were significantly different. Four variants were observed significance association after adjusting for sex, ethnicity and age in the logistic regression, including $\operatorname{rs780668}(\mathrm{P}=0.001)$, rs3764147 $(\mathrm{P}<$ $0.001)$ and rs9302752 $(\mathrm{P}<0.001)$.

\section{Association Between Leprosy Clinical Phenotypes and SNPs}

After stratification of the patients according to the age at diagnosis into $\leq 14$-year and $>14$-year groups, we found that the risk allele frequency of $\mathrm{rs} 142179458$ at $H I F 1 A$ was associated with patients diagnosed at the age 14-year. (OR $=2.65,95 \% \mathrm{CI}: 1.338-5.247, \mathrm{P}=0.004)$. After adjusting for sex and ethnicity, the frequency of rs142179458 still differs between the two groups. $(\mathrm{OR}=2.313,95 \% \mathrm{CI}$ : 1.158-4.621, $\mathrm{P}=0.02$ ) (S5 Table).

According to the bacillary load, we divided the patients into multibacillary leprosy (MB) and paucibacillary leprosy (PB) groups, ${ }^{22}$ the frequencies of alleles did not differ between the two groups, as well as the logistic regression (S6 Table). After stratification of the patients into tuberculoid leprosy (BT/TT) and the lepromatous leprosy (BL/LL) according to the R-J classification, ${ }^{23}$ we evaluated the association between the SNPs and the clinical classifications of leprosy. Leprosy patients carrying $\mathrm{G}$ allele of the rs3764147 or C allele of the rs9302752 had higher risk to present lepromatous leprosy than tuberculoid leprosy. However, after adjusting for age, sex and ethnicity, only rs3764147 showed the significant association between the two groups $(\mathrm{OR}=1.51,95 \% \mathrm{CI}$ : $1.26-$ $1.81, \mathrm{P}<0.001)$ (S7 Table).

We evaluated the association between the SNPs and the type of reactive reaction presented by the patients. In the $\mathrm{BB}, \mathrm{BT}$ and $\mathrm{BL}$ leprosy individuals, the rs73058713 A allele and rs3764147 A allele were overrepresented in RR-affected patients compared with RRfree patients $(\mathrm{OR}=1.69,95 \% \mathrm{CI}: 1.04-2.76, \mathrm{P}=0.03$; 
Table I General Characteristics of the Study Groups

\begin{tabular}{|c|c|c|c|c|c|c|c|}
\hline \multirow[t]{2}{*}{ Variables } & \multirow{2}{*}{$\begin{array}{l}\text { Leprosy } \\
(n=1344)\end{array}$} & \multirow{2}{*}{$\begin{array}{l}\text { Controls }(n=2732) \\
\text { Heredity-Related } \\
\text { Contacts }(n=1908)\end{array}$} & \multirow[t]{2}{*}{ P-value } & \multirow{2}{*}{$\begin{array}{l}\text { First - Degree } \\
\text { Family Members } \\
(n=\mid 560)\end{array}$} & \multirow[t]{2}{*}{ P-value } & \multirow{2}{*}{$\begin{array}{l}\text { Non-Heredity- } \\
\text { Related Contacts } \\
(n=824)\end{array}$} & \multirow[t]{2}{*}{ P-value } \\
\hline & & & & & & & \\
\hline Age (year, mean $\pm S D$ ) & $44.05 \pm 15.80$ & $34.17 \pm 15.54$ & $<0.001$ & $34.84 \pm 15.60$ & $<0.001$ & $46.57 \pm|2.8|$ & $<0.001$ \\
\hline $\begin{array}{l}\text { Age at diagnosis (year, } \\
\text { mean } \pm S D \text { ) }\end{array}$ & $41.35 \pm 15.69$ & & & & & & \\
\hline $\begin{array}{l}\text { Sex } \\
\qquad \text { Male } \\
\text { Female }\end{array}$ & $\begin{array}{l}936(69.6) \\
408(30.4)\end{array}$ & $\begin{array}{l}I 123(58.9) \\
785(4 I .1)\end{array}$ & $<0.001$ & $\begin{array}{l}918(58.8) \\
642(41.2)\end{array}$ & $<0.001$ & $\begin{array}{l}248(30.1) \\
576(69.9)\end{array}$ & $<0.001$ \\
\hline $\begin{array}{l}\text { Ethnicity } \\
\text { Minority } \\
\text { Han }\end{array}$ & $\begin{array}{l}633(47.1) \\
711(52.9)\end{array}$ & $\begin{array}{l}802(42.0) \\
1106(58.0)\end{array}$ & 0.004 & $\begin{array}{l}688(55.9) \\
872(44.1)\end{array}$ & 0.106 & $\begin{array}{l}37 I(45.0) \\
453(55.0)\end{array}$ & 0.347 \\
\hline $\begin{array}{l}\text { Onset age } \\
\leq 14 Y \\
>14 Y\end{array}$ & $\begin{array}{l}44(3.3) \\
1300(96.7)\end{array}$ & & & & & & \\
\hline $\begin{array}{l}\text { R-J Classification } \\
\text { Tuberculoid (BT/TT) } \\
\text { Lepromatous (LL/BL) } \\
\text { Other leprosy } \\
\text { clinical forms }\end{array}$ & $\begin{array}{l}391(29.1) \\
84 I(62.6) \\
112(8.3)\end{array}$ & & & & & & \\
\hline $\begin{array}{l}\text { WHO Classification } \\
\text { MB } \\
\text { PB }\end{array}$ & $\begin{array}{l}117 \mid(87.1) \\
173(\mid 2.9)\end{array}$ & & & & & & \\
\hline $\begin{array}{l}\text { BB, BT and BL } \\
\text { RR } \\
\text { No RR }\end{array}$ & $\begin{array}{l}54(7.2) \\
696(92.8)\end{array}$ & & & & & & \\
\hline $\begin{array}{l}\text { BL and LL } \\
\text { ENL } \\
\text { No ENL }\end{array}$ & $\begin{array}{l}75(9.5) \\
713(90.5)\end{array}$ & & & & & & \\
\hline $\begin{array}{l}\text { Reactional leprosy } \\
\text { RR } \\
\text { ENL } \\
\text { RR+ENL }\end{array}$ & $\begin{array}{l}67(41.6) \\
79(49.1) \\
15(9.3)\end{array}$ & & & & & & \\
\hline
\end{tabular}

Abbreviations: R-J Classification, Ridley-Jopling classification; TT, tuberculoid leprosy; BT, borderline tuberculoid leprosy; BL, borderline lepromatous leprosy; LL, lepromatous leprosy; PB, paucibacillary leprosy; MB, multibacillary leprosy; RR, reversal reaction; ENL, erythema nodosum leprosum.

$\mathrm{OR}=1.85,95 \%$ CI: $1.19-2.87, \mathrm{P}=0.007$ in the logistic regression, respectively), while the $\mathrm{rs} 3764147 \mathrm{G}$ allele was overrepresented in BL and LL leprosy patients who showed ENL-affected compared to the free of ENL patients $(\mathrm{OR}=1.44,95 \% \mathrm{CI}: 1.02-2.04, \mathrm{P}=0.04$ in the logistic regression), as well as the logistic regression. Furthermore, leprosy patients carrying the rs3764147 $\mathrm{G}$ allele had a higher risk to present ENL than patients with $\mathrm{RR}(\mathrm{OR}=2.75,95 \% \mathrm{CI}: 1.65-4.59, \mathrm{P}<0.0001$ in the logistic regression) ( $\underline{\mathrm{S}}, \underline{\mathrm{S} 9}, \underline{\mathrm{S} 10}$ Tables).

\section{Discussion}

Leprosy is an infectious disease caused by M. leprae, and the disease risk may be a result of the joint effect of genotype and environmental exposure. ${ }^{24}$ With the advances in molecular biology, numerous studies have supported that specific genes and SNPs play important roles in leprosy susceptibility, its clinical forms and reactional states. ${ }^{25}$ In this context, we performed a genetic epidemiology study to evaluate the allele frequencies of 17 independent reported leprosy-relate SNP loci based on 


\begin{tabular}{|c|c|c|c|c|c|c|c|c|c|c|c|c|}
\hline 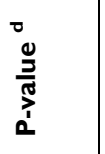 & $\stackrel{\circ}{\circ}$ & 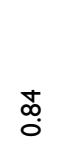 & $\stackrel{m}{0}$ & ํํㅇ & $\stackrel{\infty}{0}$ & 苞 & 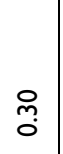 & $\frac{\text { 응 }}{0}$ & $\stackrel{\text { ֻ̊ }}{0}$ & $\bar{o}$ & $\bar{ָ}$ & $\begin{array}{l}\frac{m}{1} \\
\frac{0}{x} \\
\stackrel{0}{0} \\
\alpha\end{array}$ \\
\hline 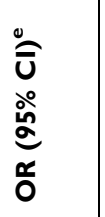 & 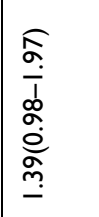 & 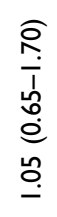 & 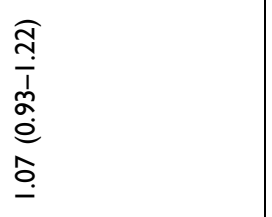 & 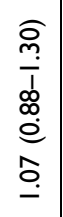 & 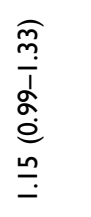 & $\begin{array}{l}\widehat{\widehat{N}} \\
\overline{1} \\
\frac{1}{\alpha} \\
\stackrel{0}{0} \\
\underline{0} \\
\underline{-}\end{array}$ & 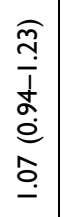 & 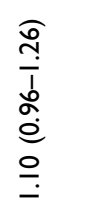 & 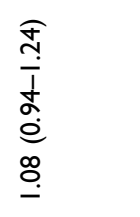 & 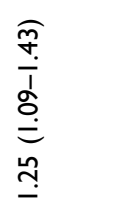 & $\begin{array}{l}\widehat{o} \\
\frac{m}{1} \\
\vdots \\
\alpha \\
0 \\
\overline{0} \\
=\end{array}$ & 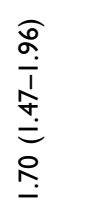 \\
\hline 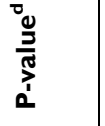 & $\stackrel{\substack{0 \\
0}}{0}$ & นิํํ & $\stackrel{\infty}{\circ}$ & స్తి & $\stackrel{\stackrel{n}{m}}{0}$ & $\frac{\overline{0}}{0}$ & $\bar{o}$ & $\stackrel{\simeq}{\circ}$ & $\frac{\sim}{0}$ & ঃั & 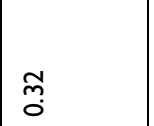 & 足 \\
\hline 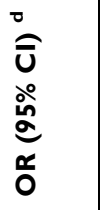 & 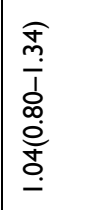 & 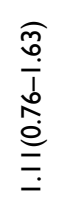 & 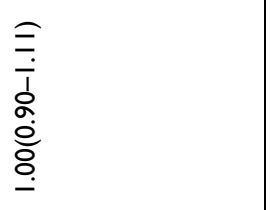 & 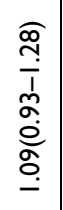 & $\begin{array}{l}\frac{\sigma}{\sigma} \\
\bar{j} \\
\alpha \\
\stackrel{0}{0} \\
\stackrel{0}{0}\end{array}$ & 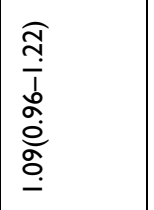 & $\begin{array}{l}\frac{\sigma}{\sigma} \\
\bar{b} \\
\circ \\
\hat{0} \\
\hat{0} \\
\dot{0}\end{array}$ & 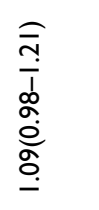 & 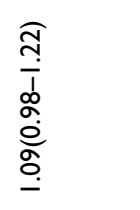 & 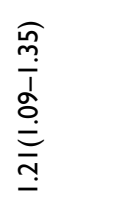 & 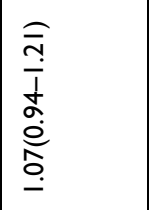 & 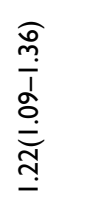 \\
\hline 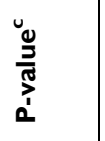 & 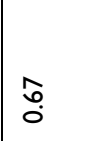 & $\underset{0}{ \pm}$ & $\stackrel{\circ}{\circ}$ & 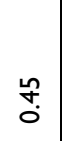 & ণ্ণি & ઼ָ & $\stackrel{\check{n}}{0}$ & 능 & $\overline{\overline{0}}$ & ఫ̊ & ণ্ণ & $\begin{array}{l}n \\
\frac{0}{x} \\
8 \\
\dot{+}\end{array}$ \\
\hline  & 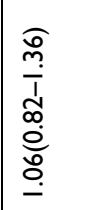 &  & 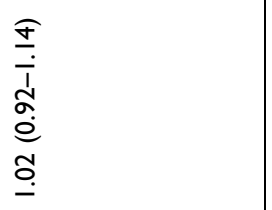 & 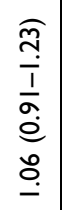 &  &  &  & 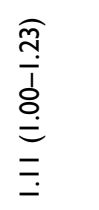 &  & 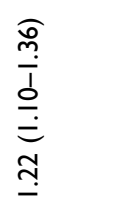 & 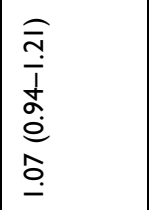 & 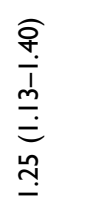 \\
\hline  & $\underset{\sim}{\tilde{O}}$ & : & นิํํ & 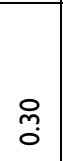 & $\frac{m}{0}$ & ઼ָ & $\overline{\bar{o}}$ & ָ̊ & $\frac{0}{0}$ & $\begin{array}{l}\stackrel{0}{0} \\
\frac{0}{x} \\
\alpha \\
\sigma \\
\sigma\end{array}$ & $\frac{0}{0}$ & $\begin{array}{l}\stackrel{0}{1} \\
\frac{0}{x} \\
\stackrel{n}{N} \\
\end{array}$ \\
\hline 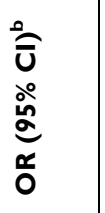 & 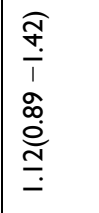 & 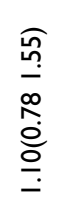 & 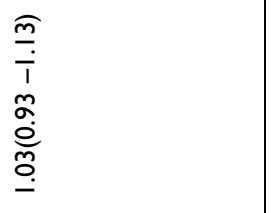 & 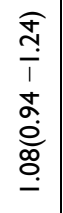 & 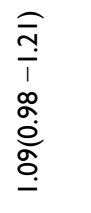 & 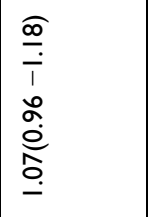 & 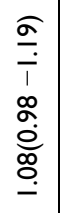 &  & 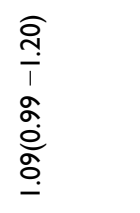 &  & 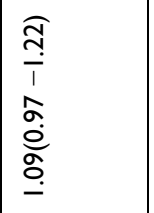 & 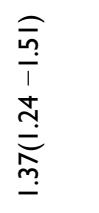 \\
\hline  & $\varangle$ & $\varangle$ & $\vdash$ & $\varangle$ & $\varangle$ & $\vdash$ & $u$ & $u$ & $u$ & $\varangle$ & $\varangle$ & $\cup$ \\
\hline 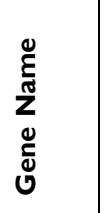 & 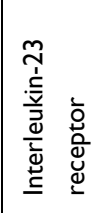 & 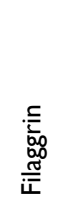 & 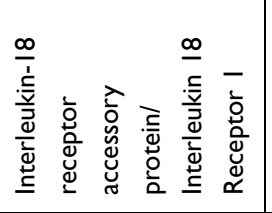 & 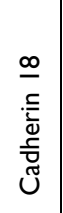 & 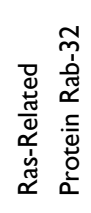 & 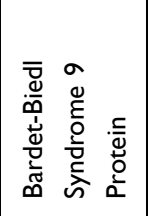 & 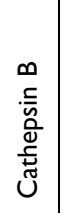 & 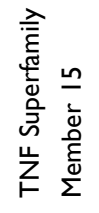 & 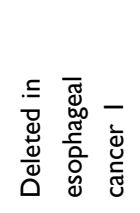 & 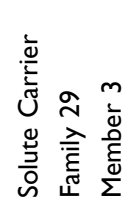 & 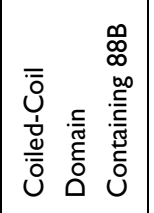 & 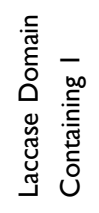 \\
\hline 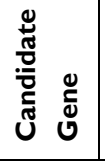 & $\stackrel{\propto}{\cong}$ & $\underset{ن}{ن}$ & 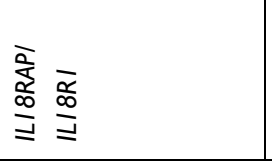 & $\frac{\infty}{\underline{I}}$ & $\begin{array}{l}\widetilde{N} \\
\tilde{a} \\
ָ\end{array}$ & $\begin{array}{l}\text { ⿵人 } \\
\text { ळ. }\end{array}$ & $\stackrel{\mathscr{ٌ}}{\iota}$ & $\frac{n}{\frac{4}{4}}$ & 苂 & 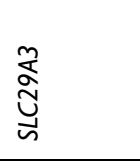 & 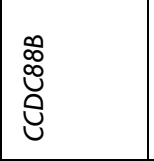 & $\overline{\breve{u}}$ \\
\hline$z_{n}$ & 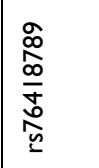 & 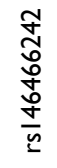 & 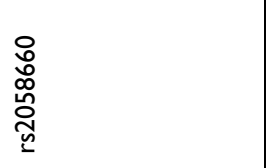 & 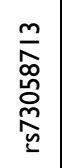 & 总 & 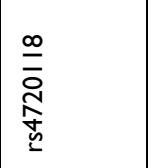 & 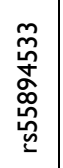 & 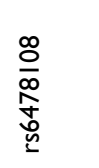 & 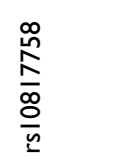 & $\begin{array}{l}\infty \\
\stackrel{0}{0} \\
\stackrel{0}{0} \\
\stackrel{0}{\underline{n}}\end{array}$ & 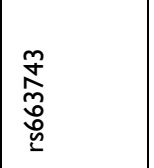 & $\begin{array}{l}\text { f } \\
\frac{+}{0} \\
\stackrel{0}{0}\end{array}$ \\
\hline
\end{tabular}




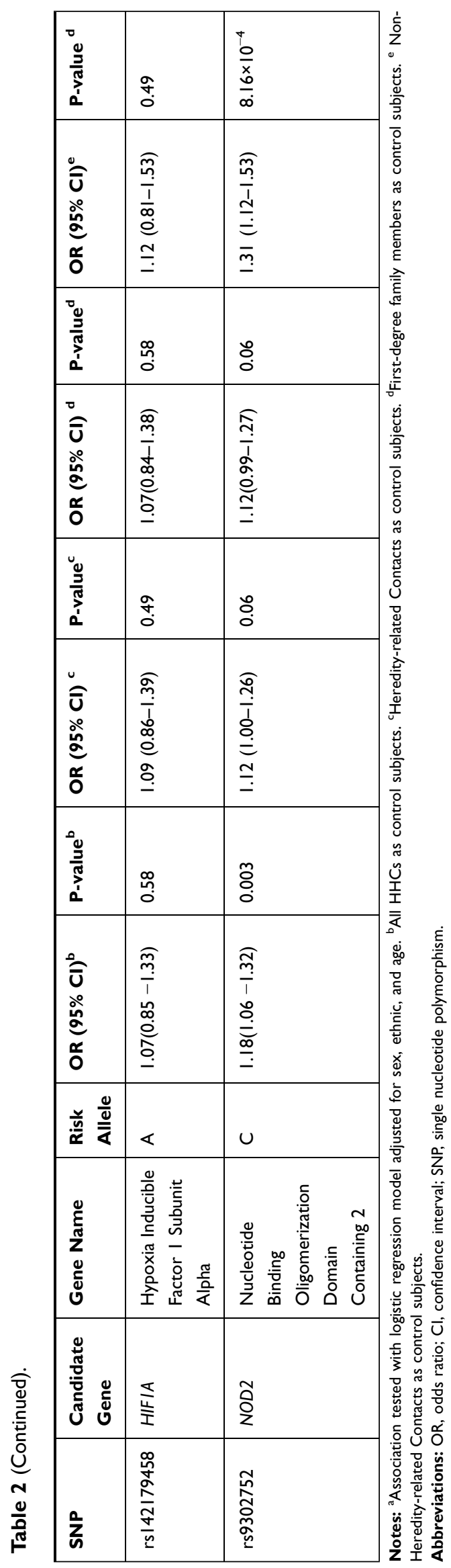

GWAS and one candidate-gene study in Chinese population. Our findings indicate that gene polymorphisms were associated with susceptibility to leprosy per se, its clinical phenotypes and reactional states.

Like many genetic-association studies, our study may be affected by the control group stratification. To address this issue, we stratified the HHCs group according to their genetic relationship to the patients and performed analysis within each group. Comparing with the all HHCs, rs780668, rs6478108, rs9302752 and rs3764147 showed significant positive correlations with leprosy after adjusting the confounding factors. However, comparing with the HHCs with no genetic relationship to the patients, rs780668 A allele, rs3764147 G allele, rs9302752 $\mathrm{C}$ allele showed significant positive correlations with leprosy even after adjusting the confounding factors. The allele distribution of rs9302752 showed no difference between patients and healthy relatives or first-degree family members control groups, and only two variants (rs780668 and rs3764147) showed significant positive association in both groups after adjusting the confounding factors. We found that significant associations of SNPs were different among the different groups of kinship, suggesting that population stratification may have been a major issue. The discovery of susceptibility genetic variant polymorphisms in the $L A C C 1$ and SLC29A3 genes has facilitated the potential application of genetic risk models, which could guide clinical professionals to formulate strategies for following up leprosy contact individuals.

Considering only leprosy patients, we found an association of the rs142179458 $\mathrm{A} / \mathrm{G}$ polymorphism at the HIF1A locus with children cases. The rs142179458 A allele at HIF1A gene had over two times higher risk to leprosy at an earlier age than leprosy in adults. Although previous studies have shown associations between susceptibility to leprosy types and polymorphisms in different genes, ${ }^{8,9}$ our data suggested that there is no significant association between PB and MB. However, rs3764147 A allele at $L A C C 1$ gene had higher risk to present tuberculoid leprosy than lepromatous leprosy $(\mathrm{OR}=1.51,95 \% \mathrm{CI}: 1.26-1.81, \mathrm{P}<$ 0.0001). We observed that exhibiting the rs73058713 A allele at the $C D H 18$ locus and rs3764147 A allele at $L A C C 1$ gene presented leprosy patients with reversal reaction. However, a greater proportion of rs $3764147 \mathrm{G}$ allele at LACC1 gene appears in ENL patients compared to BL and LL leprosy cases free of reaction and RR leprosy cases. Our data indicate that clinical workers should pay more attention 
to the patients' genetic polymorphisms in the $L A C C 1$ and $\mathrm{CDH} 18$ genes associated with leprosy reactions.

In our study, a consistent association with leprosy per se was observed for SLC29A3 and LACC1 in all HHC groups, similar to previous studies. ${ }^{8-10,18,26}$ The SLC29A3 gene encodes equilibrative nucleoside transporter 3 (ENT3), which is found in intracellular membranes, especially in lysosomal and mitochondrial membranes. Mutations in SLC29A3 could cause H Syndrome, which is rare autoinflammatory syndrome with pleiotropic manifestations. ${ }^{27}$ Previous study suspects that the ENT3 protein is highly expressed in peripheral $\mathrm{T}$ cells and has a key role in maintaining $\mathrm{T}$ cell homeostasis by supporting the proliferation and survival of T cells. ${ }^{28}$ Our data provide evidence for the possibility of ENT3 affect the final outcome of the response towards an infecting pathogen by influencing the host's immunologic status. SNP rs3764147 in $L A C C 1$ shows significant association with leprosy susceptibility and its reactive status in our study. $L A C C 1$ encodes the fatty acid metabolism-immune nexus (FAMIN) protein and was reported associations with other autoimmunity and granulomatous disease diseases,${ }^{29,30}$ which could regulate PPAR and NOD2 pathway and increase innate receptor-induced responses. FAMIN loss-of-function mutants lead to detrimental effects on the function of M0, M1 and M2 macrophages. ${ }^{31-33}$ LACC1 expression by myeloid cells in mice is essential to clear bacteria and to regulate adaptive T-cell responses against microbes. ${ }^{34}$

Our results suggested that $H I F 1 A$ is associated with onset early cases. HIFIA plays a role in immune reactions and chaperone-mediated autophagy. ${ }^{35-37}$ Increased oxidative stress has been observed in leprosy, ${ }^{38}$ and activation of the oxidative stress pathway under infection could induce HIF1A expression. ${ }^{39}$ Additionally, HIF $1 \alpha$ is a key reprogrammer of metabolism in inflammatory cells that promotes inflammatory gene expression. It is critical for the maturation of dendritic cells, activation of T cells, and drives Th17 differentiation. ${ }^{40}$ However, how this risk allele of HIFlA contributed to the early onset risk to leprosy remains to be investigated. rs73058713 at the $C D H 18$ locus was found to be associated with reversal reaction in our study, the inflammation associated with reversal reactions can lead to severe nerve injury with subsequent paralysis and deformity. Previous study suspects that $\mathrm{CDH} 18$ gene may involve in neuronal development and govern metabolic processes in later life. ${ }^{41}$ Our data reinforce the notion that leprosy reversal reaction is a neurological disease.
The strength of the study was that our study subjects were close contacts of leprosy, and subjects with or without genetically relationship of patients were studied separately, which leads to a more profound clinical significance compared with general population. And we investigated 17 SNPs association with leprosy per se and leprosy subtypes risk, the conclusions revealed by our integrative analyses were credible because of the adequacy of information on leprosy. There were a few limitations in our study. Firstly, there are some other SNPs in these genes studied in our research showed positive associations with leprosy. Moreover, the genetic variants in our study were all identified in the Chinese population. Our findings may not be extended to other ethnic populations. As a result, it might be necessary to study more polymorphisms associated with leprosy in the future. Thirdly, the retrospective design in the analysis of leprosy reactions was based on clinical grounds during the study phase and lack of longterm follow-up results, which may lead to potential bias, it might be necessary to prolong the follow-up time to detect more leprosy reaction cases in the future.

\section{Conclusion}

We demonstrated that inflammatory-related genetic variants in the LACC1, HIF1A, SLC29A3 and CDH18 genes were positively correlated with leprosy risk and leprosy clinical phenotypes in the Chinese population. These findings may provide valuable insights into further understanding the interactive role of host genetic factors in the etiology of leprosy and its clinical forms, meanwhile, this study has a potential role in finding predictive marker of leprosy development for risk stratification.

\section{Ethics Approval and Informed Consent}

This study was approved by the institutional ethical committee of the Institute of Dermatology, Chinese Academy of Medical Sciences and Peking Union Medical College, Yunnan Center for Disease Control and Prevention, Guizhou Center for Disease Control and Prevention, Hunan Center for Disease Control and Prevention, Sichuan Academy of Medical Sciences and Sichuan Provincial People's Hospital (2014-KY-003). This study was conducted according to the Declaration of Helsinki. All adult participants provided written informed consent. Parents or guardians provided written informed consent on behalf of children who participated in the study. 


\section{Acknowledgment}

We thank all the staff who have been involved in the generation of data at each local institute.

\section{Funding}

This work was supported by the Ministry of Health of China-research special funds for public health projects [grant number: 201502008], National Science and Technology Major Project [2018ZX10101-001], Chinese Academy of Medical Sciences Innovation Fund for Medical Science [2016-I2M-1-005, 2017-I2M-B\&R-14], Jiangsu Provincial Science and Technology Project [BE2018619], The Nanjing Incubation Program for National Clinical Research Center [2019060001], National Key Research and Development Program [2019YFE0113300].

\section{Disclosure}

All authors declare that they have no conflicts of interest.

\section{References}

1. Chokkakula S, Shui T, Jiang H, et al. Genotyping of Mycobacterium leprae for understanding the distribution and transmission of leprosy in endemic provinces of China. Int J Infect Dis. 2020;98:6-13. doi:10.1016/j.ijid.2020.06.032

2. Joyce MP. Historic aspects of human susceptibility to leprosy and the risk of conjugal transmission. Mem Inst Oswaldo Cruz. 2012;107 (Suppl 1):17-21. doi:10.1590/S0074-02762012000900004

3. Cambri G, Mira MT. Genetic Susceptibility to Leprosy-From Classic Immune-Related Candidate Genes to Hypothesis-Free, Whole Genome Approaches. Front Immunol. 2018;9:1674. doi:10.3389/ fimmu.2018.01674

4. Moet FJ, Pahan D, Schuring RP, Oskam L, Richardus JH. Physical distance, genetic relationship, age, and leprosy classification are independent risk factors for leprosy in contacts of patients with leprosy. J Infect Dis. 2006;193(3):346-353. doi:10.1086/499278

5. Alter A, Alcaïs A, Abel L, Schurr E. Leprosy as a genetic model for susceptibility to common infectious diseases. Hum Genet. 2008;123 (3):227-235. doi:10.1007/s00439-008-0474-z

6. Fava V, Orlova M, Cobat A, Alcaïs A, Mira M, Schurr E. Genetics of leprosy reactions: an overview. Mem Inst Oswaldo Cruz. 2012;107 (Suppl 1):132-142. doi:10.1590/S0074-02762012000900020

7. Polycarpou A, Walker SL, Lockwood DN. A Systematic Review of Immunological Studies of Erythema Nodosum Leprosum. Front Immunol. 2017;8:233. doi:10.3389/fimmu.2017.00233

8. Zhang FR, Huang W, Chen SM, et al. Genomewide association study of leprosy. $N$ Engl J Med. 2009;361(27):2609-2618. doi:10.1056/ NEJMoa0903753

9. Wang D, Fan Y, Malhi M, et al. Missense Variants in HIF1A and LACC1 Contribute to Leprosy Risk in Han Chinese. Am J Hum Genet. 2018;102(5):794-805. doi:10.1016/j.ajhg.2018.03.006

10. Liu H, Wang Z, Li Y, et al. Genome-Wide Analysis of Protein-Coding Variants in Leprosy. J Invest Dermatol. 2017;137 (12):2544-2551. doi:10.1016/j.jid.2017.08.004

11. Wang Z, Sun Y, Fu X, et al. A large-scale genome-wide association and meta-analysis identified four novel susceptibility loci for leprosy. Nat Commun. 2016;7:13760. doi:10.1038/ncomms 13760
12. Liu H, Irwanto A, Fu X, et al. Discovery of six new susceptibility loci and analysis of pleiotropic effects in leprosy. Nat Genet. 2015;47 (3):267-271. doi:10.1038/ng.3212

13. Liu H, Irwanto A, Tian H, et al. Identification of IL18RAP/IL18R1 and IL12B as leprosy risk genes demonstrates shared pathogenesis between inflammation and infectious diseases. Am J Hum Genet. 2012;91(5):935-941. doi:10.1016/j.ajhg.2012.09.010

14. Zhang F, Liu H, Chen S, et al. Identification of two new loci at IL23R and RAB32 that influence susceptibility to leprosy. Nat Genet. 2011;43(12):1247-1251. doi:10.1038/ng.973

15. Berrington WR, Macdonald M, Khadge S, et al. Common polymorphisms in the NOD2 gene region are associated with leprosy and its reactive states. $J$ Infect Dis. 2010;201(9):1422-1435. doi: $10.1086 / 651559$

16. Wong SH, Hill AV, Vannberg FO. Genomewide association study of leprosy. $N$ Engl J Med. 2010;362(15):1446-47, 1447-48.

17. Cobat A, Abel L, Alcaïs A, Schurr E. A general efficient and flexible approach for genome-wide association analyses of imputed genotypes in family-based designs. Genet Epidemiol. 2014;38 (6):560-571. doi:10.1002/gepi.21842

18. Grant AV, Alter A, Huong NT, et al. Crohn's disease susceptibility genes are associated with leprosy in the Vietnamese population. J Infect Dis. 2012;206(11):1763-1767. doi:10.1093/infdis/jis588

19. Li GD, Wang D, Zhang DF, et al. Fine mapping of the GWAS loci identifies SLC35D1 and IL23R as potential risk genes for leprosy. J Dermatol Sci. 2016;84(3):322-329. doi:10.1016/j.jdermsci.2016. 09.018

20. Li H, Durbin R. Fast and accurate short read alignment with Burrows-Wheeler transform. Bioinformatics. 2009;25(14):17 54-1760. doi:10.1093/bioinformatics/btp324

21. McKenna A, Hanna M, Banks E, et al. The Genome Analysis Toolkit: a MapReduce framework for analyzing next-generation DNA sequencing data. Genome Res. 2010;20(9):1297-1303.

22. Guidelines for the Diagnosis, Treatment and Prevention of Leprosy. Available from: https://apps.who.int/iris/bitstream/handle/10665/ 274127/9789290226383-eng.pdf?sequence=58\&isAllowed=y. Accessed May 20, 2021.

23. Ridley DS, Jopling WH. Classification of leprosy according to immunity. A five-group system. Int J Lepr Other Mycobact Dis. 1966;34 (3):255-273.

24. Yang Q, Khoury MJ. Evolving methods in genetic epidemiology. III. Gene-environment interaction in epidemiologic research. Epidemiol Rev. 1997;19(1):33-43. doi:10.1093/oxfordjournals.epirev.a017944

25. Dallmann-Sauer M, Correa-Macedo W, Schurr E. Human genetics of mycobacterial disease. Mamm Genome. 2018;29(7-8):523-538. doi:10.1186/s12881-019-0879-7

26. Xiong JH, Mao C, Sha XW, et al. Association between genetic variants in NOD2, C13orf31, and $\mathrm{CCDC} 122$ genes and leprosy among the Chinese Yi population. Int $J$ Dermatol. 2016;55 (1):65-69. doi:10.1111/ijd.12981

27. Noavar S, Behroozi S, Tatarcheh T, Parvini F, Foroutan M, Fahimi H. A novel homozygous frame-shift mutation in the SLC29A3 gene: a new case report and review of literature. BMC Med Genet. 2019;20(1):147.

28. Wei CW, Lee CY, Lee DJ, et al. Equilibrative Nucleoside Transporter 3 Regulates T Cell Homeostasis by Coordinating Lysosomal Function with Nucleoside Availability. Cell Rep. 2018;23(8):2330-2341. doi:10.1016/j.celrep.2018.04.077

29. Jostins L, Ripke S, Weersma RK, et al. Host-microbe interactions have shaped the genetic architecture of inflammatory bowel disease. Nature. 2012;491(7422):119-124. doi:10.1038/nature11582

30. Patel N, El MM, Al-Mayouf SM, et al. Study of Mendelian forms of Crohn's disease in Saudi Arabia reveals novel risk loci and alleles. Gut. 2014;63(11):1831-1832. doi:10.1136/gutjnl-2014-307859

31. Assadi G, Vesterlund L, Bonfiglio F, et al. Functional Analyses of the Crohn's Disease Risk Gene LACC1. PLoS One. 2016;11(12): e168276. doi:10.1371/journal.pone.0168276 
32. Cader MZ, Boroviak K, Zhang Q, et al. C13orf31 (FAMIN) is a central regulator of immunometabolic function. Nat Immunol. 2016;17(9):1046-1056. doi:10.1038/ni.3532

33. Lahiri A, Hedl M, Yan J, Abraham C. Human LACC1 increases innate receptor-induced responses and a LACC1 disease-risk variant modulates these outcomes. Nat Commun. 2017;8:15614. doi:10.1038/ ncomms 15614

34. Kang JW, Yan J, Ranjan K, Zhang X, Turner JR, Abraham C. Myeloid Cell Expression of LACC1 Is Required for Bacterial Clearance and Control of Intestinal Inflammation. Gastroenterology. 2020;159(3):1051-1067. doi:10.1053/j.gastro.2020.07.024

35. Hellwig-Bürgel T, Stiehl DP, Wagner AE, Metzen E, Jelkmann W. Review: hypoxia-inducible factor-1 (HIF-1): a novel transcription factor in immune reactions. J Interferon Cytokine Res. 2005;25 (6):297-310. doi:10.1089/jir.2005.25.297

36. Metheni M, Lombès A, Bouillaud F, Batteux F, Langsley G. HIF-1 $\alpha$ induction, proliferation and glycolysis of Theileria-infected leukocytes. Cell Microbiol. 2015;17(4):467-472. doi:10.1111/cmi.12421
37. Ferreira JV, Fôfo H, Bejarano E, et al. STUB1/CHIP is required for HIF1A degradation by chaperone-mediated autophagy. Autophagy. 2013;9(9):1349-1366. doi:10.4161/auto.25190

38. Jyothi P, Riyaz N, Nandakumar G, Binitha MP. A study of oxidative stress in paucibacillary and multibacillary leprosy. Indian J Dermatol Venereol Leprol. 2008;74(1):80. doi:10.4103/0378-6323.38428

39. Movafagh S, Crook S, Vo K. Regulation of hypoxia-inducible factor-1a by reactive oxygen species: new developments in an old debate. J Cell Biochem. 2015;116(5):696-703. doi:10.1002/jcb.25074

40. Corcoran SE, O'Neill LA. HIF1 $\alpha$ and metabolic reprogramming in inflammation. J Clin Invest. 2016;126(10):3699-3707. doi:10.1172/ JCI84431

41. Schlicht K, Nyczka P, Caliebe A, et al. The metabolic network coherence of human transcriptomes is associated with genetic variation at the cadherin 18 locus. Hum Genet. 2019;138(4):375-388. doi:10.1007/s00439-019-01994-x

\section{Publish your work in this journal}

Pharmacogenomics and Personalized Medicine is an international, peer-reviewed, open access journal characterizing the influence of genotype on pharmacology leading to the development of personalized treatment programs and individualized drug selection for improved safety, efficacy and sustainability. This journal is indexed on the American Chemical Society's Chemical Abstracts Service (CAS). The manuscript management system is completely online and includes a very quick and fair peer-review system, which is all easy to use. Visit http://www.dovepress.com/testimonials.php to read real quotes from published authors. 